\section{Mortalidade por doença meningocócica no Município de São Paulo, Brasil: características e preditores}

\author{
Mortality from meningococcal disease in the city of \\ São Paulo, Brazil: characteristics and predictors
}

\author{
Mortalidad por enfermedad meningocócica en el \\ municipio de São Paulo, Brasil: características \\ y predictores
}

\begin{abstract}
This study aimed to describe the magnitude, mortality, and case-fatality rate from meningococcal disease and to investigate predictors of death from this cause in the city of São Paulo, Brazil, from 1986 to 2004, using unconditional multiple logistic regression. We analyzed 10,087 cases of meningococcal disease in the city. Mean annual mortality was 1.0/100,000 inhabitants, ranging from 0.2 to 1.8. Case-fatality was 20.5\%, with major differences according to age, serogroup, and type of hospital. Predictors of death from meningococcal disease were age, especially the age brackets from one to two years and 40 years and older, and serogroup W. The results can contribute to the elaboration of public policies with a focus on the organization of hospital care and protocols to promote greater treatment effectiveness and application of vaccination strategies that decrease the incidence in groups at greatest risk of death from meningococcal disease.
\end{abstract}

Meningococcal Infections; Lethality; Mortality
Eliana Tiemi Masuda 1

Telma Regina Marques Pinto Carvalhanas 2 Rachel Maria Borelli Paradella Fernandes 3 Silvana Tadeu Casagrande ${ }^{+}$

Paula Suzuki Okada 1

Eliseu Alves Waldman 1

\section{Resumo}

O estudo objetiva descrever a magnitude, as características da mortalidade e da letalidade por doença meningocócica e investigar preditores de óbito por essa causa, no Município de São Paulo, Brasil, de 1986 a 2004. Utilizou-se a regressão logística múltipla não condicional para a investigação dos preditores de óbitos. Foram estudados 10.087 casos de doença meningocócica no município. A taxa anual média de mortalidade foi de 1,0/100 mil habitantes/ano, variando de 0,2 a 1,8; a letalidade foi de 20,5\% com grandes diferenças segundo idade, sorogrupo e tipo de hospital. Os preditores de óbito por doença meningocócica foram idade, especialmente as faixas etárias de um a dois anos e de 40 anos ou mais e o sorogrupo $W$. Os resultados obtidos podem contribuir para a elaboração de políticas públicas com foco na organização da assistência hospitalar e elaboração de protocolos que promovam a maior efetividade do tratamento $e$ a aplicação de estratégias de vacinação que diminuam a incidência nos grupos de maior risco para óbito por doença meningocócica.

Infecções Meningocócicas; Letalidade; Mortalidade 


\section{Introdução}

A doença meningocócica é uma das mais graves emergências médicas, cujo prognóstico está, em boa parte, condicionado ao diagnóstico e ao tratamento precoce 1 . A letalidade se situa entre $9 \%$ e $12 \%$ em países desenvolvidos, sendo mais elevada em países em desenvolvimento ${ }^{2}$. As sequelas acometem de $11 \%$ a $19 \%$ dos sobreviventes 3 . As crianças menores de cinco anos apresentam maior risco deóbito, enquantoaletalidadesemostra mais elevada nas faixas etárias extremas 1,4,5.

Os determinantes das formas graves da doença meningocócica são ainda insuficientemente conhecidos, porém se aceita que sejam influenciados pela virulência do patógeno e características do hospedeiro $1,6,7$, por outro lado, a mortalidade é também influenciada pelas condições de vida e de acesso a serviços de saúde 8,9.

No Brasil, dados recentes apontaram uma letalidade média em torno dos $20 \% 2,10$, acima do encontrado em outros países latino-americanos 2. Alguns autores sugerem que esse nível elevado encontrado no Brasil esteja associado não só a fatores biológicos e sociais, mas também às características da assistência médica 1,7. Pesquisas feitas em países desenvolvidos também apontam a influência da qualidade da assistência médica 11,12 , no entanto esse aspecto tem sido pouco estudado.

A doença meningocócica é endêmica nas grandes metrópoles brasileiras 2,4,13. O Município de São Paulo sofre frequentes ondas epidêmicas, não raramente é observada a sobreposição de epidemias de dois sorogrupos. Na década de 1970, por exemplo, o Município de São Paulo vivenciou epidemias simultâneas pelos sorogrupos A e C 14, e na de 1990, pelos sorogrupos B e C, atingindo taxas de incidência de 8 e de mortalidade de 1,5/100 mil habitantes/ano ${ }^{4}$.

Considerando os elevados níveis de mortalidade e letalidade associados à doença meningocócica no Município de São Paulo, o fato de essa doença atingir segmentos mais vulneráveis da população ${ }^{4}$, sobretudo as crianças, sendo a terceira causa de óbito entre menores de cinco anos nesse município (Rede Intergerencial de Informações para a Saúde. http://tabnet2.datasus. gov.br/cgi/tabcgi.exe?idb2012/c17.def, acessado em 02/Fev/2014) e também o pequeno número de estudos a respeito em grandes metrópoles brasileiras 1,7 , desenvolveu-se a presente pesquisa com o objetivo de descrever e analisar a magnitude e as características da mortalidade e da letalidade pela doença meningocócica no Município de São Paulo e investigar preditores de óbito por essa causa.

\section{Métodos}

Trata-se de estudo de corte transversal com um componente descritivo e outro analítico. A área de interesse foi o Município de São Paulo, com população estimada de 11 milhões de habitantes (Instituto Brasileiro de Geografia e Estatística. 13o Recenseamento Geral do Brasil. http://www.ibge. gov.br/home/estatistica/populacao/censo2010/ sinopse/sinopse_tab_uf_pdf.shtm, acessado em 02/Fev/2014) e dividido em 96 distritos administrativos. Possui boa infraestrutura urbana e indicadores socioeconômicos favoráveis; seu Índice de Desenvolvimento Humano (IDH) é de 0,841, mas varia de 0,245 a 0,884 , conforme o distrito, o que expressa as amplas disparidades sociais existentes 15 . A população de estudo inclui indivíduos residentes no Município de São Paulo que apresentaram doença meningocócica entre 1986 e 2004.

Foram definidos como caso indivíduos residentes no Município de São Paulo que, entre 1986 e 2004, apresentaram qualquer das formas clínicas da doença meningocócica invasiva (meningite, meningite com meningoccemia e meningoccemia) com diagnóstico etiológico em amostras de sangue ou líquor confirmado por bacterioscopia, cultura e testes indiretos de contraimunoeletroforese ou ainda, por critérios clínico-epidemiológicos, mediante achados de necropsia com necrose aguda de suprarrenal compatível com síndrome de Waterhouse-Friederichsen, ou por quadro de purpura fulminans, associado ou não à meningite.

Foram incluídos no estudo somente os casos notificados à vigilância passiva da doença meningocócica do Município de São Paulo e que cumpriam a definição de caso. Por sua vez, foram excluídos os casos atendidos e notificados por serviços de saúde do Município de São Paulo, mas residentes em outros municípios e os casos de meningites bacterianas que não a meningocócica.

As fontes de dados utilizadas foram: (i) ficha de notificação de meningites (aspectos clínicolaboratorais e epidemiológicos): Centro de Vigilância Epidemiológica Professor Alexandre Vranjac (CVE), da Secretaria de Estado da Saúde de São Paulo; (ii) dados laboratoriais: Instituto Adolfo Lutz de São Paulo (IAL)/Laboratório de Referência Nacional para Meningites Bacterianas (sorogrupos das cepas de Neisseria meningitidis); e (iii) dados populacionais: Fundação Sistema Estadual de Análise de Dados (Fundação Seade).

As bases de dados de 1986 a 1997 foram digitadas, ano a ano, e armazenadas eletronicamente no formato Dbase (dbf), pela equipe do CVE, valendo-se da ficha de notificação. De 1998 a 2004, foram digitadas pelas unidades notifican- 
tes e armazenados dados eletronicamente na versão SINAN (Sistema de Informação de Agravos de Notificação) Windows. Para a preparação do banco único para todo o período do estudo, elaborou-se previamente um dicionário de variáveis para cada uma das versões; em seguida, as variáveis foram padronizadas separadamente e, por fim, efetuou-se a junção das duas bases de dados em uma única.

Previamente à análise dos dados, foram eliminadas as duplicidades mediante pareamento dos nomes do paciente, data de nascimento, endereço de residência e nome da mãe; logo após se verificou a consistência dos dados. As formas clínicas e a confirmação do caso como doença meningocócica foram revisadas com fundamento nas variáveis relativas a sintomas e sinais e nos resultados de laboratório existentes nas fichas de notificação (1986-1997) na base de dados SINAN (1998-2004). Depois, procedeu-se à análise com o software estatístico SPSS versão 20.0 (IBM Corp., Armonk, Estados Unidos).

\section{Análise dos dados}

As variáveis analisadas foram: sociodemográficas (sexo, idade, IDH do distrito de residência); relativas ao agente (sorogrupo); formas clínicas; características da assistência hospitalar (tipo de hospital, tempo de evolução clínica do início dos sintomas até a internação) e aspectos relativos ao tempo (ano de ocorrência eperíodos epidêmicos).

Foram analisados os dados por sorogrupo da $N$. meningitidis para os casos confirmados por cultura, contraimunoeletroforese e aglutinação em látex. Para análise segundo distrito de residência no Município de São Paulo, foram utilizados os dados a partir de 1997, pois a partir deste ano até 2004 não houve alteração na divisão administrativa do Município de São Paulo. Para as características dos serviços, os hospitais foram classificados em: (i) Hospital Especializado - inclui o único hospital de referência para doenças infecciosas do Município de São Paulo; (ii) Hospitais Universitários - abrange seis unidades pertencentes a instituições acadêmicas; e (iii) Hospitais Gerais -194 hospitais públicos e privados que não se enquadram nos dois grupos anteriores.

Inicialmente, foram descritos os resultados, usando para as comparações de variáveis categóricas o teste do qui-quadrado e o teste exato de Fischer, ou ainda o qui-quadrado de tendência, quando os dados sugerissem tendência linear; para variáveis contínuas o teste de Student ou o de Whitney-Wilcoxon.

Para as estimativas das taxas de incidência, foram utilizados como numerador os casos confirmados de doença meningocócica e como de- nominador a população para o meio do período. Para estimar as taxas de mortalidade, tomou-se o número de óbitos por doença meningocócica como numerador e a população para o meio do período como denominador. Por sua vez, a letalidade foi estimada com os óbitos como numerador e o total de casos de doença meningocócica como denominador. Foram usadas razões de taxas (RT) para comparações das taxas de mortalidade. A descrição do comportamento da doença meningocócica tentará identificar ondas epidêmicas e períodos interepidêmicos, apontando características específicas de cada período.

Para a investigação de preditores de gravidade da doença meningocócica, analisaram-se os casos ocorridos entre 1997 a 2004, tomando o óbito como variável dependente; como variáveis independentes, os fatores biológicos e socioambientais e institucionais, ajustados para sexo e ano calendário, considerando comportamento epidêmico/endêmico da doença meningocócica. As estimativas das odds ratio (OR) não ajustadas e ajustadas e dos respectivos intervalos de $95 \%$ de confiança (IC95\%) foram efetuadas por meio de regressão logística múltipla não condicional. Utilizou-se o processo de stepwise forward para a entrada das variáveis no modelo. Foram selecionadas para o modelo de regressão logística múltipla as variáveis que se mostraram significantes na análise bivariada, com um valor de $\mathrm{p}<0,20 \mathrm{e}$ aquelas com plausibilidade biológica. A significância estatística das variáveis nos modelos foi avaliada pelo teste de razão de verossimilhança.

Esta pesquisa foi aprovada pelo Comitê de Ética da Faculdade de Saúde Pública da Universidade de São Paulo (protocolo de pesquisa 1772/2008).

\section{Resultados}

De 1986 a 2004, 10.087 casos de doença meningocócica invasiva foram notificados em residentes no Município de São Paulo e cumpriam os critérios de inclusão na pesquisa. Desse total, $34,4 \%$ foram confirmados pela cultura, $13,0 \%$ por técnicas de imunodiagnóstico, $19,8 \%$ por bacterioscopia, $2,6 \%$ pela necropsia e $30,2 \%$ pelo critério clínico. Não havia informação do desfecho (alta ou óbito) em 9,7\% (981/10.087) dos casos. As informações relativas ao sorogrupo estavam presentes em 46,2\% (4.648/10.087) dos casos, no entanto essa proporção foi de $27,0 \%$ para os casos que evoluíram para óbito, e de $51,1 \%$ dos que se curaram.

Dos 10.087 casos de doença meningocócica, 53,8\% eram homens, 39,8\% eram menores de dois anos, a média de idade foi de 9,3 anos e 
mediana de quatro anos. A proporção de sequelas foi 5,1\% (259/5.041), excluído o período de 1998 a 2001 cuja informação não se encontrava disponível. Em 80,7\% (209/259) dos casos estavam disponíveis informações sobre o tipo de sequela. As mais frequentes foram: necrose de tecido com $18,2 \%$, seguidas pela coleção subdural com 12,0\%, 10,0\% de hipoacusia, 9,6\% de ataxia, $8,1 \%$ de hidrocefalia e $4,3 \%$ de necrose com amputação.

\section{Incidência}

No período de 1986 a 2004, a taxa de incidência anual média foi de 5,3/100 mil habitantes/ano, com pico de 8,1 em 1995 e mínimo de 1,2 em 1986 (Figuras 1, 2a e 2b). De 1987 a 2002, verificou-se uma onda epidêmica do sorogrupo B com picos de incidência de 1,8/100 mil habitantes/ano em 1989 e de 2,0 em 1996. Foram observadas três ondas epidêmicas associadas ao sorogrupo $\mathrm{C}$, com picos em 1991 (1,0/100 mil habitantes/ano), em 1995 (1,1/100 mil) e em 2004 (1,5/100 mil) (Figura 1). A incidência pelo sorogrupo $W$ apresentou taxa média de 0,1/100 mil habitantes/ano, com um pico de 0,2 em 1988 e 2004 (Figura 1). Vale salientar que as incidências por sorogrupo estão subestimadas pela ausência dessa informação em 53,9\% dos casos analisados.

\section{Mortalidade por doença meningocócica}

A taxa de mortalidade média anual foi de 1,0/100 mil habitantes/ano, a menor taxa ocorreu em 1986 e 1987 com 0,2 e a maior em 1995 com 1,8
(Figura 1). A taxa foi mais elevada em homens, $1,1 / 100$ mil habitantes/ano, enquanto entre as mulheres situou-se em 0,8/100 mil habitantes/ ano, com RT de 1,3 (IC95\%: 1,2-1,5) para o período (Tabela 1).

As taxas de mortalidade entre menores de um ano foram as mais elevadas, variando de 2,8 (1986-1987) a 27,8/100 mil habitantes/ano (1995-1996); seguida pelo grupo de um ano de idade com taxas variando de 2,3 (1986-1987) a 14,5/100 mil habitantes/ano (1995-1996) (Tabela 1, Figuras 2c e 2d). Tomando-se como referência as taxas de mortalidade do período pré-epidêmico (1986-1987) e comparando-as com as taxas no pico da epidemia (1995-1996), a RT foi de 7,1 para a mortalidade total (todas as faixas etárias), com destaque para os menores de um ano com RT de 9,8; 13,8 para três anos; e 8,6 para 10 a 14 anos.

Estavam disponíveis informações de sorogrupo para $27 \%$ dos óbitos (505/1.869), sendo $51,5 \%$ (260/505) associados ao sorogrupo B; $26,1 \%(132 / 505)$ ao $\mathrm{C} ; 4,8 \%(24 / 505)$ ao $\mathrm{W} ; 0,2 \%$ $(1 / 505)$ ao Y e em $17,4 \%(88 / 505)$ deles a N. meningitidis não era tipável. A média de idade dos óbitos associados ao sorogrupo W foi de 18,9 anos, sendo mais elevada do que a verificada no sorogrupo $B$, com média de 11,7 anos $(p=0,054)$, e o no C com 12,8 anos ( $\mathrm{p}=0,163)$.

Entre 1997 e 2004, período em que foi possível fazer comparações segundo distrito de residência, houve 4.142 casos e 693 óbitos; mas para $14,7 \%$ (609/4.142) dos casos não existia informação de desfecho. Daqueles que evoluíram para óbitos, $35,4 \%$ residiam em distritos de IDH entre 0,245 a 0,$449 ; 53,6 \%$ entre os residentes

Figura 1

Letalidade e taxas de incidência e de mortalidade por doença meningocócica. Município de São Paulo, Brasil, 1986-2004.

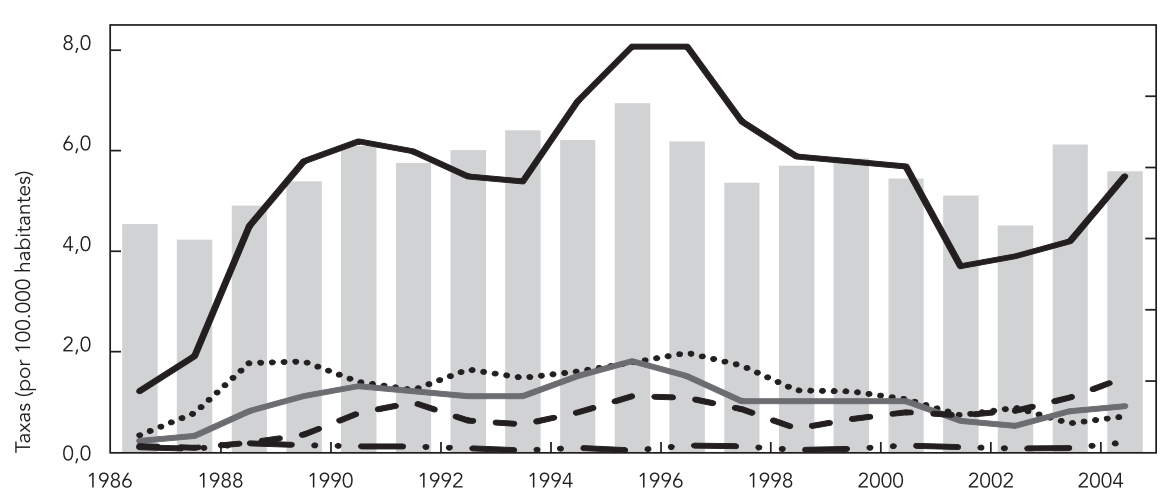

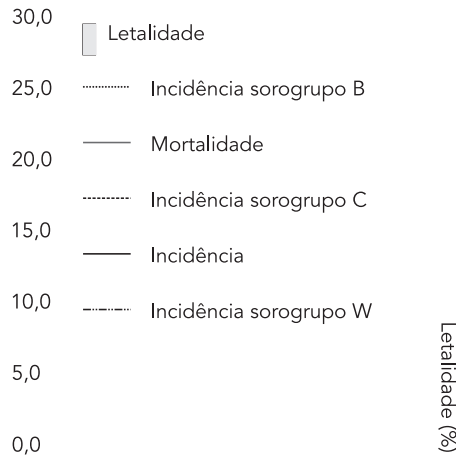

Ano de ocorrência 
Incidência (2a, 2b) e mortalidade (2c e 2d) por doença meningocócica, segundo faixa etária e taxa de incidência média anual (por 100.000 habitantes) Município de São Paulo, Brasil, período de 1986 a 2004
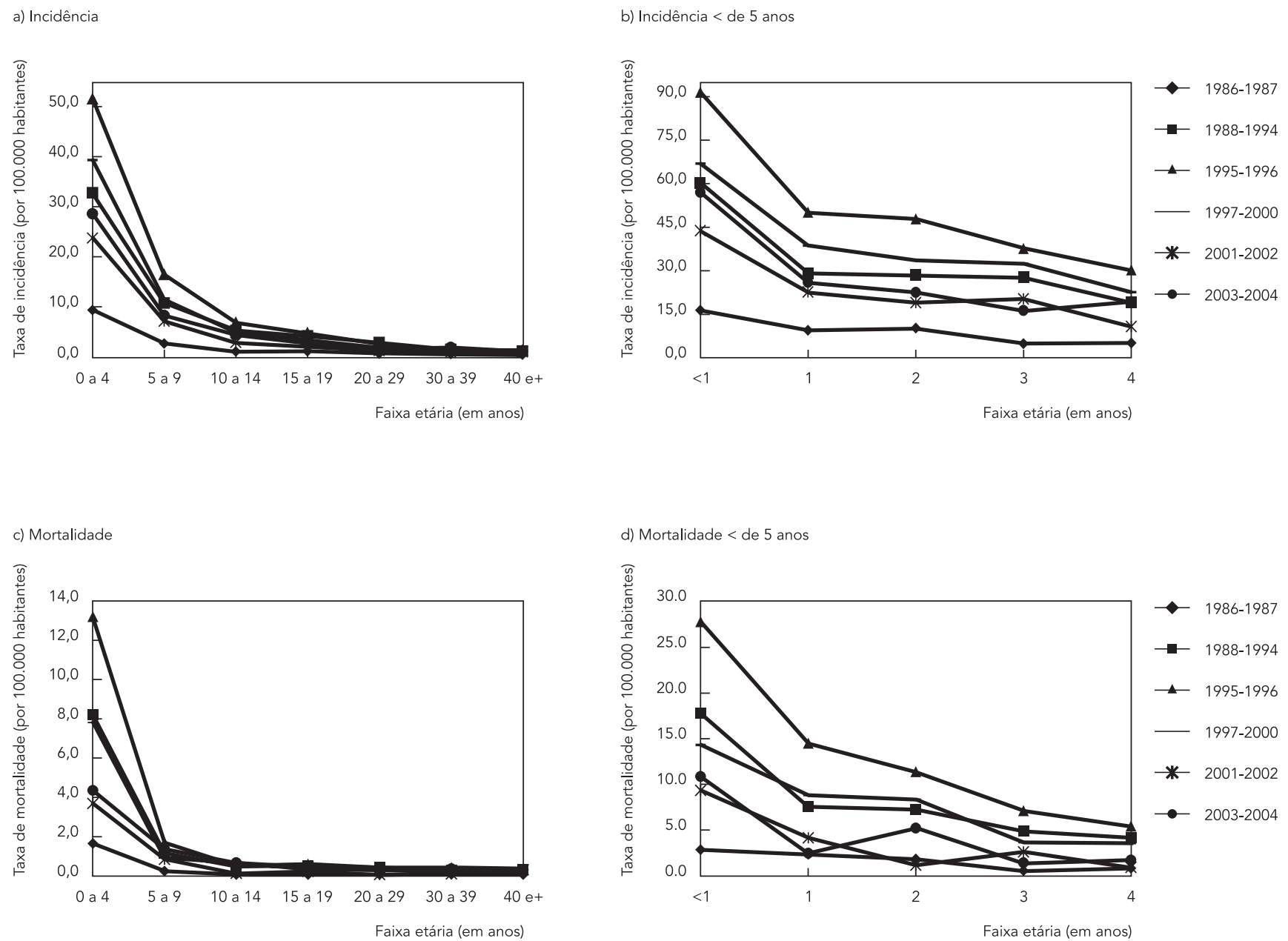

em distritos com IDH entre 0,450 a 0,$599 ; 9,3 \%$ nos distritos com esse índice entre 0,600 a 0,780 e, finalmente, $1,7 \%$ nos distritos situados entre 0,781 a 0,884 . A taxa média de mortalidade em distritos com IDH elevado $(0,781$ a 0,884$)$ foi de 0,3/100 mil habitantes/ano, elevando-se à medida que esse indicador se tornava mais desfavorável, atingindo 1,0 nos IDH entre 0,245 a 0,449 (Tabela 1).

\section{Letalidade por doença meningocócica}

A letalidade média no período foi de 20,5\% (1.869/9.106), variando de 15\%, em 1987, a 24,6\%, em 1995 (Figura 1), não sendo influenciada pela sazonalidade $(\mathrm{p}=0,995)$ (Tabela 1$)$. A letalidade entre os homens foi $21,1 \%$; entre as mulheres, de $19,8 \%(p=0,109)$ (Tabela 1). A letalidade variou com a idade, situando-se em $27,4 \%$ em menores de dois anos, atingindo $32,8 \%$ na faixa com mais de 40 anos (Tabela 1).

A letalidade variou conforme a forma clínica, sendo mais elevada nos casos de meningococcemia, atingindo $66,0 \%$ entre menores de um ano e $71,4 \%$ para 40 e mais anos; enquanto nos quadros de meningite sem meningococcemia foi mais baixa em todas as faixas etárias.

Analisando a letalidade por sorogrupo, verificou-se que foi maior nos casos associados ao sorogrupo W com $17,8 \%$, seguida pelo B com $12,1 \%$ e $11,0 \%$ no C ( $<<0,001 ;$ qui-quadrado de tendência) (Tabela 1). A letalidade entre os casos 
Tabela 1

Taxa de mortalidade * e letalidade (\%) da doença meningocócica, segundo período de ocorrência, sexo, faixa etária, forma clínica, intervalo de tempo entre o início dos sintomas até a internação, sorogrupo, Índice de Desenvolvimento Humano (IDH). Município de São Paulo, Brasil, período de $1986-2004$.

\begin{tabular}{|c|c|c|c|c|c|c|c|c|c|c|c|c|c|c|}
\hline \multirow[t]{2}{*}{ Variáveis } & \multicolumn{2}{|c|}{$\begin{array}{l}1986-1987 \\
(n=45 * \star)\end{array}$} & \multicolumn{2}{|c|}{$\begin{array}{c}1988-1994 \\
(n=797 \star \star)\end{array}$} & \multicolumn{2}{|c|}{$\begin{array}{c}1995-1996 \\
(n=334 * \star)\end{array}$} & \multicolumn{2}{|c|}{$\begin{array}{l}1997-2000 \\
(n=415 * \star)\end{array}$} & \multicolumn{2}{|c|}{$\begin{array}{c}2001-2002 \\
(n=111 \star *)\end{array}$} & \multicolumn{2}{|c|}{$\begin{array}{c}2003-2004 \\
\left(n=1677^{\star \star}\right)\end{array}$} & \multicolumn{2}{|c|}{$\begin{array}{c}\text { Total } \\
(n=1.869 * *)\end{array}$} \\
\hline & $M$ & $L$ & M & $\mathbf{L}$ & M & $\mathbf{L}$ & $M$ & $\mathbf{L}$ & $M$ & L & $M$ & $\mathbf{L}$ & $M$ & $\mathbf{L}$ \\
\hline \multicolumn{15}{|l|}{ Sexo } \\
\hline Feminino & 0,15 & 11,54 & 1,00 & 19,88 & 1,39 & 21,58 & 0,92 & 20,44 & 0,45 & 16,61 & 0,62 & 19,60 & 0,84 & 19,77 \\
\hline Masculino & 0,33 & 18,52 & 1,34 & 21,52 & 1,97 & 24,84 & 1,06 & 19,21 & 0,61 & 17,42 & 0,95 & 21,27 & 1,12 & 21,10 \\
\hline \multicolumn{15}{|l|}{ Faixa etária (anos) } \\
\hline$<1$ & 2,81 & 17,74 & 17,74 & 31,19 & 27,76 & 34,27 & 14,27 & 24,88 & 9,29 & 25,00 & 10,82 & 23,30 & 14,67 & 28,46 \\
\hline 1 & 2,30 & 24,32 & 7,52 & 26,93 & 14,45 & 31,10 & 8,80 & 25,82 & 4,10 & 19,74 & 2,41 & 11,39 & 6,99 & 25,40 \\
\hline 2 & 1,79 & 18,42 & 7,19 & 26,30 & 11,34 & 26,32 & 8,28 & 28,86 & 1,12 & 7,14 & 5,16 & 28,79 & 6,37 & 25,49 \\
\hline 3 & 0,51 & 10,53 & 4,85 & 18,56 & 7,08 & 21,01 & 3,63 & 12,69 & 2,57 & 15,00 & 1,38 & 9,80 & 3,74 & 16,41 \\
\hline 4 & 0,77 & 15,00 & 4,11 & 22,55 & 5,38 & 20,00 & 3,53 & 17,78 & 0,87 & 9,09 & 1,68 & 11,54 & 3,16 & 18,95 \\
\hline $5-9$ & 0,23 & 9,52 & 1,15 & 11,25 & 1,69 & 11,86 & 0,92 & 9,72 & 0,83 & 13,86 & 1,35 & 19,66 & 1,05 & 11,81 \\
\hline $10-14$ & 0,06 & 7,69 & 0,47 & 9,40 & 0,50 & 8,33 & 0,67 & 16,11 & 0,12 & 4,88 & 0,66 & 18,33 & 0,46 & 11,16 \\
\hline $15-19$ & 0,06 & 6,67 & 0,51 & 13,25 & 0,60 & 13,41 & 0,34 & 12,04 & 0,21 & 12,12 & 0,45 & 19,05 & 0,40 & 13,23 \\
\hline $20-29$ & 0,05 & 10,53 & 0,43 & 16,23 & 0,42 & 19,75 & 0,29 & 19,17 & 0,05 & 5,26 & 0,31 & 24,00 & 0,31 & 17,00 \\
\hline $30-39$ & 0,07 & 18,18 & 0,29 & 22,70 & 0,42 & 38,89 & 0,18 & 18,75 & 0,09 & 12,00 & 0,35 & 21,05 & 0,24 & 22,46 \\
\hline 40 e + & 0,05 & 18,75 & 0,29 & 30,94 & 0,37 & 36,84 & 0,23 & 30,11 & 0,30 & 41,67 & 0,30 & 35,59 & 0,27 & 32,82 \\
\hline \multicolumn{15}{|l|}{ Forma clínica } \\
\hline Meningite & 0,05 & 6,87 & 0,17 & 8,31 & 0,16 & 7,33 & 0,07 & 4,51 & 0,06 & 4,96 & 0,11 & 7,04 & 0,12 & 6,94 \\
\hline Meningite com & 0,09 & 12,41 & 0,45 & 15,38 & 0,69 & 16,00 & 0,19 & 7,82 & 0,11 & 8,42 & 0,23 & 13,37 & 0,32 & 13,22 \\
\hline \multicolumn{15}{|l|}{ meningococemia } \\
\hline Meningococemia & 0,10 & 51,351 & 0,54 & 59,141 & 0,83 & 50,613 & 0,75 & 39,793 & 0,36 & 33,784 & 0,44 & 36,614 & 0,54 & 46,03 \\
\hline \multicolumn{15}{|c|}{ Intervalo de tempo entre o início } \\
\hline \multicolumn{15}{|c|}{ dos sintomas até a internação } \\
\hline 2-3 dias & 0,02 & 3,16 & 0,11 & 7,46 & 0,15 & 10,03 & 0,11 & 9,16 & 0,05 & 6,51 & 0,10 & 8,71 & 0,10 & 8,05 \\
\hline 1 dia & 0,12 & 21,70 & 0,40 & 22,63 & 0,56 & 19,54 & 0,36 & 16,32 & 0,16 & 12,27 & 0,28 & 16,91 & 0,34 & 18,99 \\
\hline$<24$ horas & 0,05 & 33,33 & 0,24 & 39,27 & 0,51 & 33,66 & 0,39 & 25,67 & 0,17 & 20,81 & 0,24 & 24,41 & 0,27 & 29,72 \\
\hline \multicolumn{15}{|l|}{ Sorogrupo } \\
\hline C & 0,01 & 7,14 & 0,06 & 9,92 & 0,13 & 13,61 & 0,07 & 12,35 & 0,05 & 8,09 & 0,12 & 10,92 & 0,07 & 10,95 \\
\hline B & 0,03 & 5,15 & 0,18 & 12,35 & 0,24 & 14,72 & 0,12 & 11,21 & 0,07 & 10,71 & 0,07 & 14,42 & 0,14 & 12,14 \\
\hline W & 0,00 & 0,00 & 0,01 & 11,11 & 0,02 & 30,00 & 0,01 & 11,54 & 0,02 & 30,77 & 0,04 & 36,36 & 0,01 & 17,78 \\
\hline \multicolumn{15}{|l|}{ Sazonalidade } \\
\hline Janeiro/Março & 0,02 & 13,40 & 0,22 & 20,21 & 0,35 & 26,74 & 0,20 & 19,35 & 0,12 & 14,88 & 0,17 & 22,50 & 0,19 & 20,56 \\
\hline Abril/Junho & 0,05 & 13,89 & 0,29 & 22,02 & 0,46 & 25,56 & 0,25 & 18,81 & 0,14 & 18,63 & 0,15 & 15,46 & 0,24 & 20,71 \\
\hline Julho/Setembro & 0,11 & 20,79 & 0,36 & 20,40 & 0,53 & 22,15 & 0,27 & 18,44 & 0,17 & 18,46 & 0,24 & 23,72 & 0,30 & 20,42 \\
\hline Outubro/Dezembro & 0,06 & 11,46 & 0,29 & 20,29 & 0,34 & 19,94 & 0,29 & 23,68 & 0,09 & 15,75 & 0,23 & 21,15 & 0,24 & 20,46 \\
\hline \multicolumn{15}{|l|}{$\mathrm{IDH} * \star \star \star$} \\
\hline $0,781-0,884$ & - & - & - & - & - & - & 0,52 & 13,24 & 0,12 & 6,25 & 0,12 & 5,26 & 0,32 & 10,68 \\
\hline $0,600-0,780$ & - & - & - & - & - & - & 0,71 & 18,96 & 0,25 & 10,00 & 0,50 & 20,90 & 0,54 & 17,53 \\
\hline $0,450-0,599$ & - & - & - & - & - & - & 0,95 & 19,85 & 0,50 & 17,23 & 0,75 & 21,65 & 0,79 & 19,76 \\
\hline $0,245-0,449$ & - & - & - & - & - & - & 1,24 & 21,41 & 0,61 & 19,37 & 0,78 & 23,50 & 0,97 & 21,44 \\
\hline \multicolumn{15}{|l|}{ Tipo de hospital \# } \\
\hline Especializado & - & 8,62 & - & 12,08 & - & 13,42 & - & 11,03 & - & 4,59 & - & 12,00 & - & 11,66 \\
\hline Universitário & - & 36,36 & - & 25,91 & - & 18,59 & - & 18,46 & - & 10,96 & - & 18,18 & - & 21,11 \\
\hline Gerais & - & 45,95 & - & 33,20 & & 28,40 & - & 21,50 & - & 20,25 & - & 21,58 & - & 26,17 \\
\hline Total & 0,23 & 15,41 & 1,17 & 20,71 & 1,67 & 23,29 & 0,99 & 20,02 & 0,53 & 17,05 & 0,78 & 20,64 & 0,97 & 20,52 \\
\hline
\end{tabular}

L: letalidade; M: mortalidade.

* Taxa de mortalidade por 100 mil habitantes/ano;

** Número de óbitos;

*** IDH do Distrito de residência;

\# Tipo de hospital: a taxa de mortalidade não foi estimada em virtude de essas unidades não atenderem a uma população definida. 
associados ao sorogrupo W foi elevada em todos os grupos etários, especialmente no de 40 e mais anos com 33,3\%.

A letalidade encontrada em distritos com IDH entre 0,245 a 0,449 foi de $21,4 \%$, situandose em $19,8 \%$ nos distritos com IDH entre 0,450 e 0,599 , em $17,5 \%$ naqueles com IDH entre 0,600 a 0,780 e $10,7 \%$ naqueles entre 0,781 a 0,884 ( $\mathrm{p}=$ 0,008; qui-quadrado de tendência) (Tabela 1).

O período estudado coincide com a descentralização da assistência hospitalar no Município de São Paulo, com expressiva diminuição do atendimento dos casos de doença meningocócica no Hospital Especializado, que declinou de 79,9\%, (1986-1987) para 20,1\% (2003-2004) (Figura 3a). A distribuição média dos atendimentos dos casos de doença meningocócica no período foi de 55,3\% nos Hospitais Gerais, 35,7\% no Especializado e 9,0\% nos Universitários. Seguindo a mesma ordem, a letalidade média foi de $26,2 \%$, $11,7 \%$ e $21,1 \%$, sendo menor no Hospital Especializado se comparado aos demais $(\mathrm{p}<0,001$; qui-quadrado de tendência) (Tabela 1). A letalidade no Hospital Especializado foi constante em torno de $12 \%$, contrastando com o constatado nos outros dois tipos de hospitais onde esse indicador variou bastante, declinando no final do período (2003-2004), quando situou-se em 18,2\% no Universitário e 21,6\% nos Hospitais Gerais (Figura 3a).

Não se observaram diferenças entre as médias de idade dos óbitos ocorridos nos Hospitais Gerais (9,6 anos) e no Especializado (8,9 anos) ( $p=0,435)$, contudo a média da idade dos óbitos nos Hospitais Universitários (13,5 anos) foi mais elevada do que a verificada nos dois outros tipos de hospital $(p=0,002)$.

A maior proporção de casos com meningococcemia foi atendida pelos Hospitais Gerais, com $27,4 \%$, vs. 23,8\%, nos Hospitais Universitários e 12,5\% no Especializado ( $\mathrm{p}<0,001$; quiquadrado de tendência) (Figura $3 b$ ). A letalidade da meningococcemia se situou em $56,3 \%$ nos Hospitais Gerais, 47,3\% nos Universitários e em $36,7 \%$ no Especializado ( $p<0,001$; qui-quadrado de tendência) (Figura 3c). O tempo médio entre o início dos sintomas até a internação foi de 1,4 dia nos Hospitais Gerais, sendo menor se comparado a 2,0 dias no Especializado $(p<0,001)$ e 1,6 dia nos Universitários $(\mathrm{p}<0,05)$.

A distribuição das internações nos hospitais, segundo o IDH do distrito de residência mostrou diferenças em relação ao atendimento de pacientes residentes em distritos mais pobres, onde o acesso ao atendimento hospitalar Especializado é mais difícil. Nos Hospitais Gerais, 32,6\% dos pacientes residiam distritos com IDH entre 0,245-0,449, no Especializado essa proporção foi de 26,3\% e nos Universitários 19,9\% ( $\mathrm{p}<0,001$; qui-quadrado de tendência) (Figura 3d).

Preditores de óbito por doença meningocócica

Na investigação de preditores de óbito por doença meningocócica, na análise bivariada, mostraram-se associados ao óbito: ter menos de um ano (OR = 2,1; IC95\%: 1,6-2,7); um a dois anos (OR = 2,0; IC95\%: 1,5-2,5); 40 anos ou mais (OR = 3,4; IC95\%: 2,4-4,8); doença meningocócica pelo sorogrupo W (OR = 2,7; IC95\%: 1,4-5,0); residir em distrito com IDH entre 0,450-0,599 (OR = 2,1; IC95\%: 1,1-3,9) e em IDH entre 0,2450,449 (OR = 2,3; IC95\%: 1,2-4,3). No modelo final, após ajuste para os diferentes períodos segundo o comportamento endêmico/epidêmico da doença meningocócica e sexo, mostraram-se independentemente associados ao óbito por doença meningocócica: ter um a dois anos de idade (OR = 1,7; IC95\%: 1,1-2,8); 40 anos ou mais (OR = 2,0; IC95\%: 1,1-3,8); e doença meningocócica pelo sorogrupo W (OR = 2,7; IC95\%: 1,4-5,4) (Tabela 2).

\section{Discussão}

As elevadas taxas de morbimortalidade por doença meningocócica verificadas no Município de São Paulo apontam a carga dessa doença no município. A mortalidade é tão elevada que excede as de incidências da doença meningocócica encontradas em países desenvolvidos $16 \mathrm{e}$ em alguns países latino-americanos com estágio de desenvolvimento semelhante ao brasileiro 2 . Os dados apresentados são semelhantes aos encontrados em outras regiões do Brasil 5,7,10, o que justifica a classificação do país como uma das poucas áreas epidêmicas de doença meningocócica fora do continente africano 17.

A frequência de sequelas encontrada se situa em nível inferior ao descrito na literatura 3,18 , provavelmente em virtude da falta de completude dos dados ou ainda devido à elevada letalidade encontrada no estudo se comparada a países desenvolvidos. Entretanto, os tipos de lesões mais frequentes constituem mais um indicador da gravidade da doença meningocócica e coincidem com os resultados de outros estudos 3 .

As taxas de incidência da doença meningocócica apontam a frequência com que ocorrem as ondas epidêmicas no Município de São Paulo e os elevados patamares que alcançam, inclusive, com sobreposição de epidemias pelos sorogrupos B e C em 1995 a 1996. Apontam também algumas diferenças no comportamento dos 
Figura 3

Perfil dos hospitais de atendimento dos casos de doença meningocócica segundo distribuição percentual por tipo de hospital e letalidade. Município de São Paulo, Brasil, período de 1986 a 2004.

3a) Ano de ocorrência

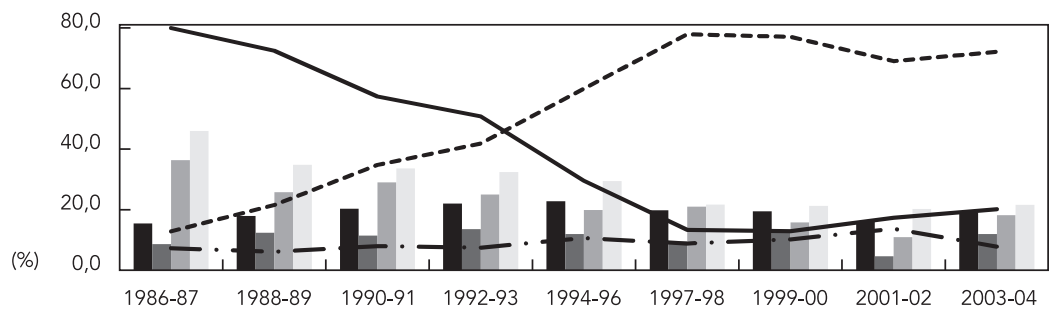

Letalidade

Município de São Paulo

Especializado

Universitários

Gerais
Internação

- Especializado

-...- Universitários

-..-........ Gerais

3b) Faixa etária (anos)

(\%)

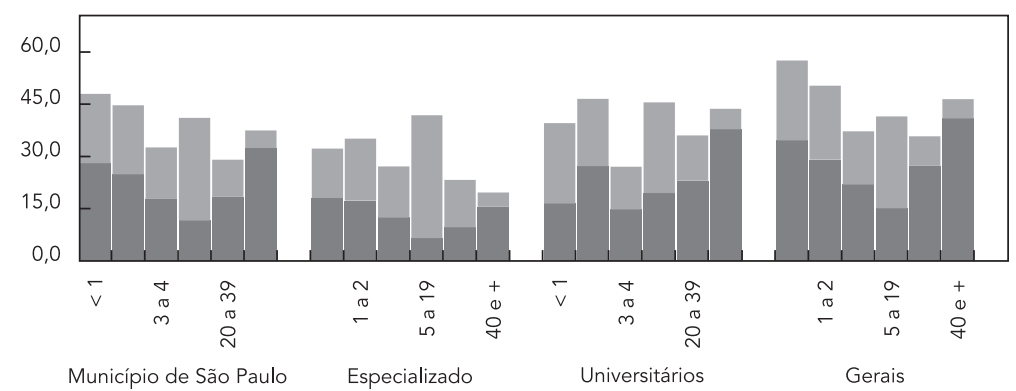

Letalidade

\% de internação

3c) Forma clínica

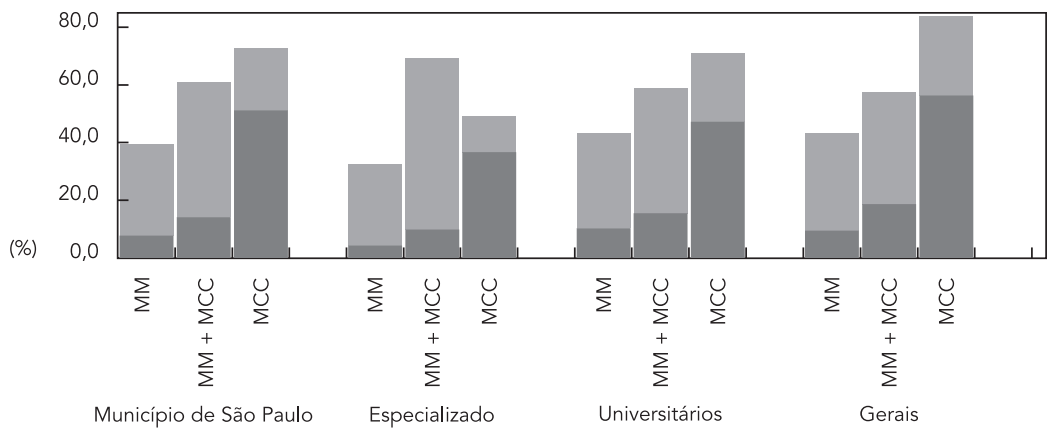

Letalidade

ـ \% de internação

3d) IDH dos distritos de residência

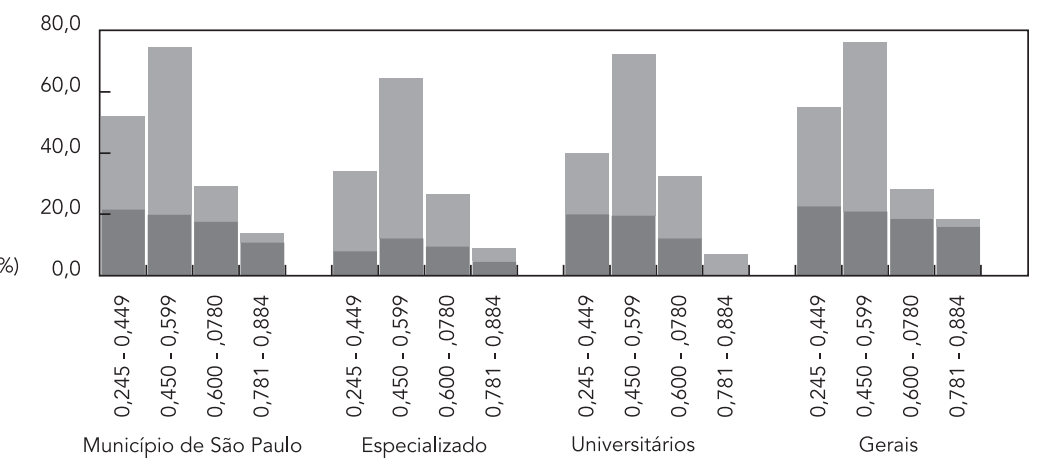

Letalidade

\% de internação

IDH: Índice de Desenvolvimento Humano; MCC: meningococcemia; MM: meningite meningocócica. 
Fatores associados ao óbito da doença meningocócica (modelo final). Município de São Paulo, Brasil, período de $1997-2004$.

\begin{tabular}{|c|c|c|c|c|c|}
\hline \multirow[t]{3}{*}{ Características } & \multicolumn{2}{|c|}{ Óbito } & \multirow[t]{3}{*}{$\mathrm{OR}_{\mathrm{B}}(\mathrm{IC} 95 \%)$} & \multirow[t]{3}{*}{$\mathrm{OR}_{\mathrm{A}}(\mathrm{IC} 95 \%)$} & \multirow[t]{3}{*}{ Valor de $p$ * } \\
\hline & Não (\%) & $\operatorname{Sim}(\%)$ & & & \\
\hline & {$[N=2.840]$} & {$[\mathrm{N}=702]$} & & & \\
\hline Período & & & & & 0,212 \\
\hline $2001-2002$ & $540(82,9)$ & $111(17,1)$ & 1,0 (Referência) & 1,0 (Referência) & \\
\hline $1997-2000$ & $1.658(80,0)$ & $415(20,0)$ & $1,2(1,0-1,5)$ & $1,2(0,8-2,0)$ & \\
\hline $2003-2004$ & $642(79,4)$ & $167(20,6)$ & $1,3(1,0-1,6)$ & $1,6(0,9-2,7)$ & \\
\hline \multicolumn{6}{|l|}{ Sexo } \\
\hline Feminino & $1.538(80,4)$ & $374(19,6)$ & 1,0 (Referência) & 1,0 (Referência) & 0,466 \\
\hline Masculino & $1.302(80,4)$ & $318(19,6)$ & $1,0(0,8-1,2)$ & $0,9(0,6-1,3)$ & \\
\hline Faixa etária (anos) & & & & & $<0,001$ \\
\hline $5-19$ & $213(85,2)$ & $37(14,8)$ & 1,0 (Referência) & 1,0 (Referência) & \\
\hline$\leq 1$ & $1.111(76,1)$ & $349(23,9)$ & $2,1(1,6-2,7)$ & $0,7(0,4-1,2)$ & \\
\hline $1-2$ & $456(86,4)$ & $72(13,6)$ & $2,0(1,5-2,5)$ & $1,7(1,1-2,8)$ & \\
\hline $1-4$ & $469(87,3)$ & $68(12,7)$ & $1,0(0,7-1,4)$ & $0,5(0,2-1,1)$ & \\
\hline $20-39$ & $171(82,2)$ & $37(17,8)$ & $1,4(1,0-2,0)$ & $0,9(0,5-1,8)$ & \\
\hline 40 ou + & $131(65,5)$ & $69(34,5)$ & $3,4(2,4-4,8)$ & $2,0(1,1-3,8)$ & \\
\hline Sorogrupo & & & & & 0,023 \\
\hline C & $550(89,1)$ & $67(10,9)$ & 1,0 (Referência) & 1,0 (Referência) & \\
\hline B & $618(88,4)$ & $81(11,6)$ & $1,1(0,8-1,5)$ & $1,1(0,8-1,6)$ & \\
\hline W & $46(75,4)$ & $15(24,6)$ & $2,7(1,4-5,0)$ & $2,7(1,4-5,4)$ & \\
\hline IDH ** & & & & & 0,672 \\
\hline $0,781-0,884$ & $92(89,3)$ & $11(10,7)$ & 1,0 (Referência) & 1,0 (Referência) & \\
\hline $0,600-0,780$ & $287(82,5)$ & $61(17,5)$ & $1,8(0,9-3,5)$ & $1,0(0,3-2,9)$ & \\
\hline $0,450-0,599$ & $1.433(80,2)$ & $353(19,8)$ & $2,1(1,1-3,9)$ & $1,4(0,5-3,6)$ & \\
\hline $0,245-0,449$ & $854(78,6)$ & $233(21,4)$ & $2,3(1,2-4,3)$ & $1,3(0,5-3,8)$ & \\
\hline
\end{tabular}

IC95\%: intervalo de 95\% de confiança; IDH: Índice de Desenvolvimento Humano; $\mathrm{OR}_{\mathrm{A}}$ : odds ratio ajustada; $\mathrm{OR}_{\mathrm{B}}$ : odds ratio bruta.

* $\mathrm{p}<0,001$ (teste da razão de verossimilhança);

** IDH do distrito de residência.

diferentes sorogrupos, por exemplo, ondas epidêmicas mais prolongadas quando associadas ao $\mathrm{B}{ }^{4}$ e graus distintos de gravidade, especialmente na doença meningocócica pelo sorogrupo W 19.

As elevadas taxas de mortalidade em menores de quatro anos de idade, particularmente entre os menores de um ano e a semelhança do encontrado na literatura, confirmam a maior vulnerabilidade desse grupo etário 1,5,6. A sobreposição de dois sorogrupos B e C deve explicar as taxas de mortalidade mais elevadas observadas no período de pico de epidemia (1995-1996).

A mortalidade é mais elevada nos distritos mais pobres, seguindo com pequena variação o comportamento da incidência que, por sua vez, é fortemente influenciada pelas condições de vida da população, particularmente as más condições habitacionais 4,5,6,14. O Município de São Paulo possui cerca de 270 mil moradias classificadas como domicílios subnormais (Fundação Seade.
Indicadores Selecionados do Município de São Paulo. http://www.seade.gov.br/produtos/msp/ tabela_sintese.htm, acessado em 01/Jul/2014). O fato de a mortalidade e a letalidade serem mais elevadas em distritos com predomínio de população pobre pode ser também explicado pelo menor acesso à assistência à saúde, tendo em vista a rápida evolução da doença meningocócica 9 .

Semelhante a outros estudos brasileiros 1,5,20, a letalidade se mostrou muito elevada, especialmente nas faixas etárias extremas. Em média, foi praticamente o dobro daquela encontrada em países desenvolvidos 16,20, onde patamares tão elevados são encontrados somente em surtos epidêmicos, geralmente associados a cepas de $N$. meningitidis mais virulentas 21 .

A letalidade se eleva no final dos anos 1980 até meados da década seguinte, o que pode ser explicado pelo fato de acompanhar a tendência da epidemia associada ao sorogrupo B, que 
mostrou letalidade mais alta do que a doença meningocócica pelo sorogrupo C. Porém, esse período coincide também com a descentralização da assistência hospitalar e a rápida diminuição da participação do Hospital Especializado e correspondente ampliação do atendimento pelos Hospitais Gerais, o que permite a hipótese de que ao menos parte da elevação da letalidade esteja associada à qualidade da assistência hospitalar, o que é consistente com várias publicações que têm apontado a influência da qualidade da assistência no desfecho de pacientes com doença meningocócica, sobretudo em quadros graves de evolução rápida ao óbito que requer diagnóstico oportuno e manejo adequado 9,11,13. Podese tomar como exemplo a diminuição da letalidade verificada no pico da epidemia de doença meningocócica no Município de São Paulo, em 1974, justificada não somente pela maior atenção da população, mas também pelo diagnóstico precoce e maior experiência dos profissionais de saúde no manejo da doença 4,22 .

Alguns aspectos podem justificar a maior letalidade encontrada nos Hospitais Gerais, entre eles, o fato de tratarem maior proporção de casos graves da doença meningocócica (meningococemia) e com menor tempo médio do início dos sintomas à internação, o que sugere uma evolução rápida e grave da doença, mas também um melhor acesso pela maior descentralização do atendimento.

Não há dúvida de que a letalidade é influenciada por vários fatores do hospedeiro e do agente, no entanto a expressiva diferença da letalidade entre os Hospitais Gerais e o Especializado em todo o período, em todas as faixas etárias e independentemente da forma clínica, sugerem que tal diferença esteja de alguma forma influenciada pelas características da assistência médica. Diferença semelhante foi registrada no Rio de Janeiro, no início da década de 1990, durante a mesma epidemia de doença meningocócica pelo sorogrupo B, quando a letalidade no hospital especializado situava-se em $9 \%$ e nos demais hospitais, em 39\% 13,23.

Analisando os preditores de óbito por doença meningocócica identificados neste estudo, verifica-se que idade e sorogrupo W mostraram-se associados independentemente das demais exposições. Dois grupos etários mostraram-se associados ao óbito, crianças entre um a dois anos de idade e adultos maiores de 40 anos de idade. Em relação às crianças, é possível que esse fato esteja relacionado, em parte, à percepção tardia pelos pais, da gravidade da doença meningocócica ou, ao estado nutricional da criança e/ou à própria resposta imune nessa faixa etária 7. Já nos adultos acima dos 40 anos, pode ser influen- ciado pela maior prevalência de comorbidades 6 , pela diminuição da resposta imunológica, pelo diagnóstico tardio em virtude da tendência de se entender esse grupo etário como baixo risco para a doença meningocócica ou, ainda, pela manifestação clínica atípica 20,24.

A identificação do sorogrupo W como preditor do óbito por doença meningocócica é consistente com a literatura que sugere que a maior gravidade associada ao sorogrupo $\mathrm{W}$ esteja relacionada ao complexo clonal ST-11, que aceitase ser mais virulento 19,25. Esse complexo clonal tem sido isolado no Brasil 26. Por outro lado, a maior gravidade da doença meningocócica por esse sorogrupo tem sido apontada em estudos realizados no Brasil e em outros países da América do Sul 2,5,27.

A interpretação dos resultados apresentados deve ter em conta algumas limitações. Entre elas o fato de terem sido utilizados dados secundários de um sistema de vigilância passivo, em que a falta de completude das informações pode induzir a vieses. Outra limitação se refere ao uso de uma definição de caso sensível e ao fato de somente um terço dos óbitos possuir informações do sorogrupo, possivelmente pela maior dificuldade de colher amostra para exames laboratoriais em casos graves. Apesar de a classificação dos hospitais em três tipologias ter permitido identificar diferenças e formular hipóteses, os Hospitais Gerais e Universitários abrangem instituições com recursos humanos, instalações e equipamentos distintos, o que torna essa classificação imperfeita.

Mesmo com as limitações apontadas, este estudo apresentou resultados consistentes com a literatura e úteis para subsidiar políticas públicas, especialmente aquelas voltadas à organização de serviços, pois salientam a necessidade de uma avaliação da qualidade do atendimento hospitalar aos casos de doença meningocócica e da elaboração de protocolos que aprimorem o diagnóstico e o tratamento desse tipo de paciente. Além de assinalar a necessidade de estudos mais aprofundados sobre a influência da qualidade da assistência na elevada letalidade da doença meningocócica no Brasil.

Por outro lado, como existem poucas publicações recentes com dados prévios à introdução da vacina conjugada contra o meningococo C, seus resultados poderão subsidiar a avaliação do impacto dessa vacina na gravidade da doença meningocócica, como sugerem publicações recentes 5,28. Com base também nos resultados apresentados, pode-se recomendar o amplo uso da técnica de PCR em tempo real, como estratégia para aprimorar os dados de vigilância, em relação à identificação dos sorogrupos. 


\section{Resumen}

Los objetivos fueron describir la magnitud y las características de la morbilidad y mortalidad por enfermedad meningocócica e investigar los predictores de muerte. Fueron estudiados 10.087 casos de enfermedad meningocócica ocurridos en São Paulo, entre 1986 y 2004. Los predictores de muerte por enfermedad meningocócica se investigaron por regresión logística no condicional. La tasa anual media de mortalidad por enfermedad meningocócica en el periodo del estudio fue de 1,0/100 mil habitantes/año, variando de 0,2 a 1,8, con distribución desigual, afectando fuertemente a los distritos más pobres. La letalidad fue de 20,5\% con grandes diferencias según edad, serogrupo y tipo de hospital. Los principales predictores de muerte fueron la edad, especialmente, menores de dos años, y serogrupo W. Los resultados pueden contribuir a la elaboración de las políticas públicas con un enfoque en la organización de la atención hospitalaria y elaboración de protocolos que promuevan una mayor eficacia del tratamiento y la aplicación de las estrategias de vacunación para reducir la incidencia de los grupos con mayor riesgo de muerte por enfermedad meningocócica.

Infecciones Meningocócicas; Letalidad; Mortalidad

\section{Referências}

1. Donalisio MRC, Kemp B, Rocha MMM, Ramalheira RMF. Letalidade na epidemiologia da doença meningocócica: estudo na região de Campinas, SP, 1993-1998. Rev Saúde Pública 2000; 34:589-95.

2. Sáfadi MAP, González-Ayala $\mathrm{S}$, Jäkel $\mathrm{A}$, Wieffer $\mathrm{H}$, Moreno C, Vyse A. The epidemiology of meningococcal disease in Latin America 1945-2010: an unpredictable and changing landscape. Epidemiol Infect 2013; 141:447-58.

3. Pace D, Pollard AJ. Meningococcal disease: clinical presentation and sequelae. Vaccine 2012; 30 Suppl 2:B3-9.

\section{Colaboradores}

E. T. Masuda e E. A. Waldman participaram da concepção do projeto, análise e interpretação dos dados, redação e da aprovação final da versão a ser publicada e foram responsáveis por todos os aspectos do trabalho na garantia da exatidão e integridade de qualquer parte da obra. T. R. M. P. Carvalhanas e P. S. Okada contribuíram com a análise e interpretação dos dados, revisão crítica relevante do conteúdo intelectual do texto e da aprovação final da versão a ser publicada. R. M. B. P. Fernandes participou da revisão crítica relevante do conteúdo intelectual do texto. S. T. Casagrande (in memoriam) participou da concepção do projeto.

\section{Agradecimentos}

Agradecemos a Maria Inês Cappelletti Gonçalves pelo apoio na preparação dos bancos de dados originais obtidos junto à vigilância da doença meningocócica. Esta pesquisa foi financiada pela FAPESP (processo: 2002/08956-1) e pelo CNPq (bolsa de mestrado; processo: $133160 / 2007-6)$. 
7. Duarte MCMB, Amorim MR, Cuevas LE, CabralFilho JE, Correia JB. Risk factors for death from meningococcal infection in Recife, Brazil. J Trop Pediatr 2005; 51:227-31.

8. Heyderman RS, Ben-Shlomo Y, Brennan CA, Somerset $\mathrm{M}$. The incidence and mortality for meningococcal disease associated with area deprivation: an ecological study of hospital episode statistics. Arch Dis Child 2004; 89:1064-8.

9. Souza SF, Costa MCN, Paim JS, Natividade MS, Pereira SM, Andrade AMS, et al. Bacterial meningitis and living conditions. Rev Soc Bras Med Trop 2012; 45:323-8.

10. Azevedo LCP, Toscano CM, Bierrenbach AL. Bacterial meningitis in Brazil: baseline epidemiologic assessment of the decade prior to the introduction of pneumococcal and meningococcal vaccines. PLoS One 2013; 8:e64524.

11. Booy R, Habibi P, Nadel S, Munter C, Britto J, Morrison $\mathrm{A}$, et al. Reduction in case fatality rate from meningococcal disease associated with improved healthcare delivery. Arch Dis Child 2001; 85: 386-90.

12. Ninis N, Phillips C, Bailey L, Pollock JI, Nadel S, Britto J, et al. The role of healthcare delivery in the outcome of meningococcal disease in children: case-control study of fatal and non-fatal cases. BMJ 2005; 330:1475.

13. Gama SGN, Marzochi KBF, Siveira Filho GB. Caracterização epidemiológica da doença meningocócica na área metropolitana do Rio de Janeiro, Brasil, 1976 a 1994. Rev Saúde Pública 1997; 31:254-62.

14. Barata RCB. Epidemia de doença meningocócica, 1970-1977: aparecimento e disseminação do processo epidêmico. Rev Saúde Pública 1988; 22:16-24.

15. Secretaria do Desenvolvimento, Trabalho e Solidariedade, Prefeitura de São Paulo. Índice de Desenvolvimento Humano, IDH: Município de São Paulo, 2002. São Paulo: Prefeitura de São Paulo; 2002.

16. Harrison LH, Trotter CL, Ramsay ME. Global epidemiology of meningococcal disease. Vaccine 2009; 27 Suppl 2:B51-63.

17. Choudhuri D, Huda T, Theodoratou E, Nair H, Zgaga L, Falconer R, et al. An evaluation of emerging vaccines for childhood meningococcal disease. BMC Public Health 2011; 11 Suppl 3:S29.

18. Kirsch EA, Barton RP, Kitchen LRN, Giroir BP. Pathophysiology, treatment and outcome of meningococcemia: a review and recent experience. Pediatr Infect Dis J 1996; 15:967-79.
19. von Gottberg A, Plessis M, Cohen C, Prentice E, Schrag S, Gouveia L, et al. Emergence of endemic serogroup W135 meningococcal disease associated with a high mortality rate in South Africa. Clin Infect Dis 2008; 46:377-86.

20. Smith I, Bjornevik AT, Augland IMB, Berstad A, Wentzel-Larsen T, Halstensen A. Variations in case fatality and fatality risk factors of meningococcal disease in Western Norway, 1985-2002. Epidemiol Infect 2006; 134:103-10.

21. Brooks R, Woods CW, Benjamin DK, Rosenstein NE. Increased case-fatality rate associated with outbreaks of Neisseria meningitidis infection, compared with sporadic meningococcal disease, in the United States, 1994-2002. Clin Infect Dis 2006; 43:49-54.

22. Iversson LB. Aspectos epidemiológicos da meningite meningocócica no município de São Paulo (Brasil) no período de 1968 a 1974. Rev Saúde Pública 1976; 10:1-16.

23. Noronha CP, Struchiner CJ, Halloran ME. Assessment of the direct effectiveness of BC meningococcal vaccine in Rio de Janeiro, Brazil: a case-control study. Int J Epidemiol 1995; 24:1050-7.

24. Iversen BG, Aavitsland P. Meningococcal disease in Norway 1992-1995. Epidemiology and fatality. Scand J Infect Dis 1996; 28:253-9.

25. Weidlich L, Baethgen LF, Mayer LW, Moraes C, Klein CC, Nunes LS, et al. High prevalence of Neisseria meningitidis hypervirulent lineages and emergence of W135:P1.5,2:ST-11 clone in Southern Brazil. J Infect 2008; 57:324-31.

26. Lemos AP, Harrison LH, Lenser M, Sacchi CT. Phenotypic and molecular characterization of invasive serogroup W135 Neisseria meningitidis strains from 1990 to 2005 in Brazil. J Infect 2010; 60: 209-17.

27. Barroso DE, Rebelo MC. Recognition of the epidemiological significance of Neisseria meningitidis capsular serogroup W135 in the Rio de Janeiro region, Brazil. Mem Inst Oswaldo Cruz 2007; 102:773-5.

28. Cardoso CW, Pinto LL, Reis MG, Flannery B, Reis JN. Impact of vaccination during an epidemic of serogroup C meningococcal disease in Salvador, Brazil. Vaccine 2012; 30:5541-6.

Recebido em 07/Fev/2014

Versão final reapresentada em 05/Ago/2014 Aprovado em 29/Ago/2014 